\title{
Designing Energy-Efficient Wireless Access Networks: LTE and LTE-Advanced
}

\author{
Margot Deruyck, Wout Joseph, Bart Lannoo, Didier Colle, and Luc Martens \\ Ghent University / iMinds, Dept. of Information Technology \\ Gaston Crommenlaan 8 box 201, B-9050 Ghent, Belgium \\ Fax: +32 933 14899, E-mail: margot.deruyck@intec.UGent.be
}

\begin{abstract}
As base stations are currently large energy consumers, it is important to investigate their energy efficiency to develop more energy-efficient wireless access networks in the future. This study investigates how energy-efficient LTEAdvanced networks can be designed compared to LTE networks. Therefore, a power consumption model is developed for LTE and LTE-Advanced macrocell and femtocell base stations, along with a suitable energy efficiency measure. The influence on the energy efficiency of three main functionalities added to LTE-Advanced is investigated: carrier aggregation, heterogeneous networks, and extended MIMO support. Our study shows that the energy efficiency can be improved up to $400 \%$ and $450 \%$ by using, respectively, carrier aggregation and MIMO. For bit rates higher than $20 \mathrm{Mbps}$, the macrocell base station is the most energyefficient. Below $20 \mathrm{Mbps}$, it depends on the bit rate.
\end{abstract}

Index Terms-energy efficiency, LTE, LTE-Advanced, femtocell, macrocell, carrier aggregation, heterogeneous networks, MIMO

\section{INTRODUCTION}

In the past few years, an extreme growth of mobile users is noticed. The global mobile phone penetration has increased from $20 \%$ in 2003 to $67 \%$ in 2009 [1]. This growth has his influence on the wireless access networks, which are already large energy consumers within ICT (Information Communication Technology). The base stations (BSs) contribute to $90 \%$ of the wireless access network's power consumption [2]. To decrease the ecological footprint of ICT, it is thus important to decrease the power consumption of these networks. Therefore, a thorough study of the BS's power consumption is necessary.

In this paper, we investigate how energy-efficient LTE (Long Term Evolution)-Advanced (Release 10) access networks can be designed in comparison to LTE (Release 8/9) networks. The influence of three main functionalities added to LTEAdvanced on energy efficiency will be investigated: carrier aggregation (CA) (to increase the bit rate), heterogeneous networks (whereby macrocell and femtocell BSs are mixed in one network), and extended support for MIMO (Multiple Input Multiple Output) (where multiple antennas are used for sending and receiving the signal). Therefore, a power consumption model for both LTE and LTE-Advanced macrocell and femtocell BSs is developed, along with a suitable energy efficiency measure.

Only a few studies focus on the energy efficiency of LTE(Advanced). In [1], [3], [4] the energy efficiency of heterogeneous LTE(-Advanced) networks is investigated. However, to the best of our knowledge, a comparison between the energy efficiency of LTE and LTE-Advanced has never been done before and neither the effect on the energy efficiency of its main functionalities.

The outline of the paper is as follows. In Section II, an introduction is given on LTE(-Advanced). In Section III, the developed power consumption model for both LTE and LTEAdvanced macrocell and femtocell BSs is discussed, and a suitable energy efficiency measure is defined. Section IV presents the results obtained with the power consumption model and compares the energy efficiency of LTE-Advanced networks. Section V provides the conclusion.

\section{LTE AND LTE-ADVANCED}

LTE, standardized by 3GPP (3rd Generation Partnership Project) is nowadays an emerging wireless access technology. Different releases have been defined in the past few years [5], each adding new features while preserving backwards compatibility. The first two releases (Release 8/9) are known as LTE [5]. Release 10 is also known as LTE-Advanced [5] which supports even higher bit rates. Note that LTE and LTEAdvanced is the same technology and LTE-Advanced is thus backwards compatible with LTE.

LTE(-Advanced) allows to adaptively change modulation (for translating the digital signal to an analogue signal that can be transmitted wirelessly), coding rate (for detection of the errors occurred due to wireless transmission), and bandwidth to enhance the channel quality. Bandwidths from roughly $1 \mathrm{MHz}$ up to $20 \mathrm{MHz}$ are supported. This bandwidth can be further extended in LTE-Advanced by CA which allows to transmit multiple LTE carriers, each with a bandwidth up to $20 \mathrm{MHz}$, in parallel to the same terminal [6]. A carrier, in this context called a component carrier, contains amongst others the data that needs to be sent.

Furthermore, the support for heterogeneous networks is further improved in LTE-Advanced [6]. LTE based heterogeneous networks are typically two-layered networks with macrocell (eNodeB) and femtocell (home-eNodeB) BSs. Although this is already supported in LTE, LTE-Advanced improves the handling of the interference between the different cells [6].

Finally, the support for MIMO is also further enhanced in LTE-Advanced up to 8 transmit antennas [6]. Here, the effect of spatial diversity (extending the range) and spatial multiplexing (extending the bit rate) on the energy efficiency is investigated. 


\section{ENERGY EFFICIENCY IN WIRELESS ACCESS NETWORKS}

Determining which BS type is the most energy-efficient is not easy as different performance parameters (bandwidth, coverage, served users, etc.) can be considered. Therefore, the energy efficiency $E E\left(\in\left[0, \infty[)\right.\right.$ is defined (in $\left(\mathrm{km}^{2}\right.$. $\mathrm{Mbps}) / \mathrm{W})$ :

$$
E E=\frac{\pi \cdot R^{2} \cdot B \cdot U}{P_{e l}}
$$

with $P_{e l}$ the BS's power consumption (in Watt), $R$ the range (in $\mathrm{km}$ ), $B$ the physical bit rate by the BS (in Mbps) and $U$ the number of served users. The higher $E E$, the more energyefficient the BS is. In literature, different measures can be found mostly taking into account either the bit rate, the covered area or the number of served users. [4], respectively [7], considers the power consumption per covered area, respectively power consumption over the capacity, which is meaningful when comparing the energy efficiency of BSs offering the same capacity, respectively the same range. In [9], [10], the power consumption per user is introduced. This measure is very meaningful for wired technologies where users sharing the same modems are offered the same range and bit rate, but is less meaningful for wireless technologies as range and bit rate can differ from BS to BS. As our definition takes all those parameters into account, the aggregated influence of all these parameters on the energy efficiency can be investigated. In the next section, it is discussed how $P_{e l}, R, B$ and $U$ are determined.

\section{A. Power consumption in a wireless access network}

1) Power consumption $P_{e l}$ of a macrocell BS: Fig. 1 shows that a macrocell BS consists of six power-consuming components [2]:

- Rectifier $(100 \mathrm{~W})$ : converts alternating current (AC) to direct current (DC).

- Digital signal processing (100 W): is concerned with the conversion of the signal to a sequence of bits or symbols and the processing of these signals.

- Transceiver $(100 \mathrm{~W})$ : responsible for transmitting and receiving the signals.

- Power amplifier $(156.3 \mathrm{~W})$ : converts the DC input power into a significant radio-frequent (RF) signal.

- Air conditioning $(225 \mathrm{~W})$ : regulates the temperature in the BS cabin.

- Backhaul $(80 \mathrm{~W})$ : responsible for the communication with the backhaul network (a microwave or fiber link).

The sum of all those components corresponds with the BS power consumption. However, in Fig. 1, some components are used multiple times and their power consumption should be multiplied by their number of occurrences. Which components and how many of them depends on two factors: the number $n_{\text {sector }}$ of sectors and the number $n_{T x}$ of transmitting antennas. The area covered by a BS is called a cell which is further divided into a number $n_{\text {sector }}$ of sectors. Each sector is covered by one antenna. For each sector, we need $n_{T x}$ rectifiers, $n_{T x}$ digital signal processing components, $n_{T x}$ transceivers and $n_{T x}$ power amplifiers. The power consumption of these
MACROCELL

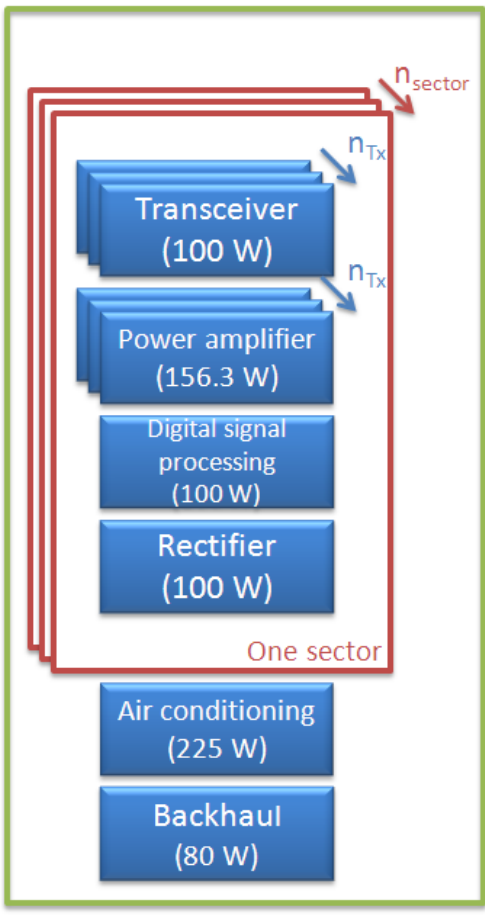

Figure 1. Block diagram of the components of a macrocell, and femtocell base station [11], [12].

components should thus be multiplied by $n_{\text {sector }}$. Unless mentioned otherwise, we assume one transmitting antenna per sector.

Each of the BS's components has its own typical power consumption (Fig. 1) which is assumed to be constant throughout time except for the air conditioning and the power amplifier. The power consumption of the latter depends on the input power $P_{T x}$ of the antenna [11]. The higher $P_{T x}$, the higher the power consumption of the power amplifier. For the macrocell BS, LTE(-Advanced) has a typical $P_{T x}$ of $43 \mathrm{dBm}$. The power consumption of the air conditioning depends on the internal and ambient temperature of the cabin which are here assumed to be $25^{\circ} \mathrm{C}$ and are thus constant.

We stated that the power consumption of each component is assumed to be constant throughout time. However, a nuance should be made. The power consumption of the digital signal processing, the transceiver and the power amplifier can fluctuate during time due to variations in load on the BS which represents the number of active users and the requirements of the used services in the BS cell. The higher the load, the higher the BS's power consumption. To take this into account, we define the load factor as a value between $0(=$ no traffic) and 1 (= maximum traffic). The power consumption of the digital signal processing, the transceiver and the power amplifier should be multiplied by this load factor to determine the BS's power consumption at a certain time of the day and varies between 0.93 and 1 during the day [2]. Here, we assume a load factor of 1 (= maximal power consumption of BS). The worst case scenario is thus investigated.

Accounting for this, we obtained a power consumption of 
$1672.6 \mathrm{~W}$ per BS (LTE and LTE-Advanced, $n_{T x}=1$ ) [2].

2) Power consumption $P_{\text {el }}$ of a femtocell BS: The size of a femtocell BS is much smaller than the one of a macrocell BS and is comparable to that of a WiFi access point. Therefore, the power-consuming components are different from those of a macrocell BS as shown in Fig. 1 [12], [13]:

- Microprocessor $(3.2 \mathrm{~W})$ : responsible for implementing and managing the standardized radio protocol stack, the baseband processing and backhauling.

- FPGA (Field-Programmable Gate Array) (4.7 W): responsible for a number of features such as data encryption, hardware authentication, etc.

- Transceiver $(1.8 \mathrm{~W})$

- Power amplifier $(2.4 \mathrm{~W})$

The power consumption of these components is indicated in Fig. 1, resulting in a femtocell BS power consumption of $12 \mathrm{~W}$ $\left(n_{T x}=1\right)$.

\section{$B$. Range of the different BS types}

To determine the BS's range, the maximum allowable path loss $P L_{\max }$ needs to be calculated. Path loss is the ratio of the transmitted power to the received power of the signal [11]. $P L_{\max }$ is then the maximum allowable path loss to which a transmitted signal can be subjected while still being detectable at the receiver.

Based on $P L_{\max }$, we can determine the range by using a propagation model which describes the relation between path loss and range. We are obliged to use a different propagation model for the two BS types as the circumstances for each type are different. E.g., the femtocell BS is placed indoor while the macrocell BS is installed outdoor, also the antenna height is different for both of them. The Erceg C and ITU-R P.1238 model are chosen for the macrocell and femtocell BS, respectively.

An important parameter is the receiver Signal-to-Noise Ratio (SNR) which represents the SNR at the receiver for a certain Bit Error Rate (BER) and depends on the modulation and coding rate used. The combination of the modulation scheme with the coding rate determines the physical bit rate which is the total number of physically transferred bits per second including useful data as well as the protocol overhead. The higher the bit rate, the higher the receiver SNR and the shorter the range is.

\section{Bit rate}

The bit rate is determined by taking into account a number of parameters such as the number of carriers that contain user data, the total number of carriers, the bandwidth, the modulation, the coding rate, and if applicable the CA. In this study only aggregation of component carriers with the same bandwidth are considered.

Note that the offered bit rate does not differ between the macrocell and femtocell BS as the same technology is considered for both BS types, they will both offering the same bit rates.

\section{Users}

Another important difference between the macrocell and femtocell BS is the maximum number of served users.

The smallest unit to which user traffic can be allocated is the so-called physical resource block (PRB) which consists of 12 carriers. For each bandwidth, the number of used carriers will be divided by 12 to determine the number of PRBs. Furthermore, we assume that each PRB is used by a different user. For the macrocell BS, this results in a maximum of $18,36,75,150,225$, and 300 users, for a bandwidth of respectively $1.4,3,5,10,15$, and $20 \mathrm{MHz}$.

For a femtocell BS, the number of users is typically limited to 16 (independent of the bandwidth) [13] and will thus be used for the numerical results.

\section{RESUlTS}

\section{A. Carrier aggregation (CA)}

In this section, it is investigated how the energy efficiency is influenced by adding CA. Fig. 2 shows the EE for some modulation schemes supported by LTE(-Advanced) for both macrocell and femtocell BSs in a $5 \mathrm{MHz}$ channel. Note that the energy efficiencies in Fig. 2 indicated as LTE apply to Release 8/9, while all these energy efficiencies are applicable to Release 10 . We also indicated how much carriers are aggregated for LTE-Advanced.

To obtain the results in Fig. 2, the power consumption, the range, and the bit rate are determined as discussed in Section III-A, III-B, and III-C, respectively. The number of served users is fixed as only one bandwidth (i.e., $5 \mathrm{MHz}$ ) is considered (Section III-D). The EE is then calculated by using eq. (1).

For each option in Fig. 2, e.g. for Macro LTE or Macro LTE-Adv. CA $2 \times 5 \mathrm{MHz}$, a higher modulation scheme and/or coding rate results in a lower EE (e.g. for a LTE-Advanced macrocell $\mathrm{BS}$ with a $\mathrm{CA}$ of two component carriers, $E E=$ $0.5\left(\mathrm{~km}^{2} \cdot \mathrm{Mbps}\right) / \mathrm{W}$ for a coding rate of $2 / 3$ and a modulation of QPSK i.e., 2/3 QPSK versus $0.2\left(\mathrm{~km}^{2} \cdot \mathrm{Mbps}\right) / \mathrm{W}$ for $2 / 364-$ QAM) because a higher modulation scheme and coding rate lead to a shorter range for a higher bit rate [2]. The decrease in range is higher than the increase in bit rate, leading to a lower $\mathrm{EE}$ as the power consumption and the number of served users remain the same.

When comparing LTE and LTE-Advanced, Fig. 2 shows that higher bit rates can be obtained even for higher EE by using CA. E.g., for a macrocell BS and 1/2 QPSK, $E E$ $=0.4\left(\mathrm{~km}^{2} \cdot \mathrm{Mbps}\right) / \mathrm{W}$ for LTE versus $2.1\left(\mathrm{~km}^{2} \cdot \mathrm{Mbps}\right) / \mathrm{W}$ when aggregating 5 component carriers of $5 \mathrm{MHz}$. This is due to the fact that CA does not influence the obtained range nor the number of served users. Introducing $\mathrm{CA}$ has little impact on the BS's power consumption because CA corresponds in practice to a multicarrier (not aggregated) configuration which is already supported by LTE or to a BS supporting multiple frequency bands depending on which type of CA is considered [6]. The extra power consumed for processing will thus be negligible compared to the BS's power consumption. It is concluded that in terms of EE, it might be interesting to immediately implement LTE-Advanced in the 


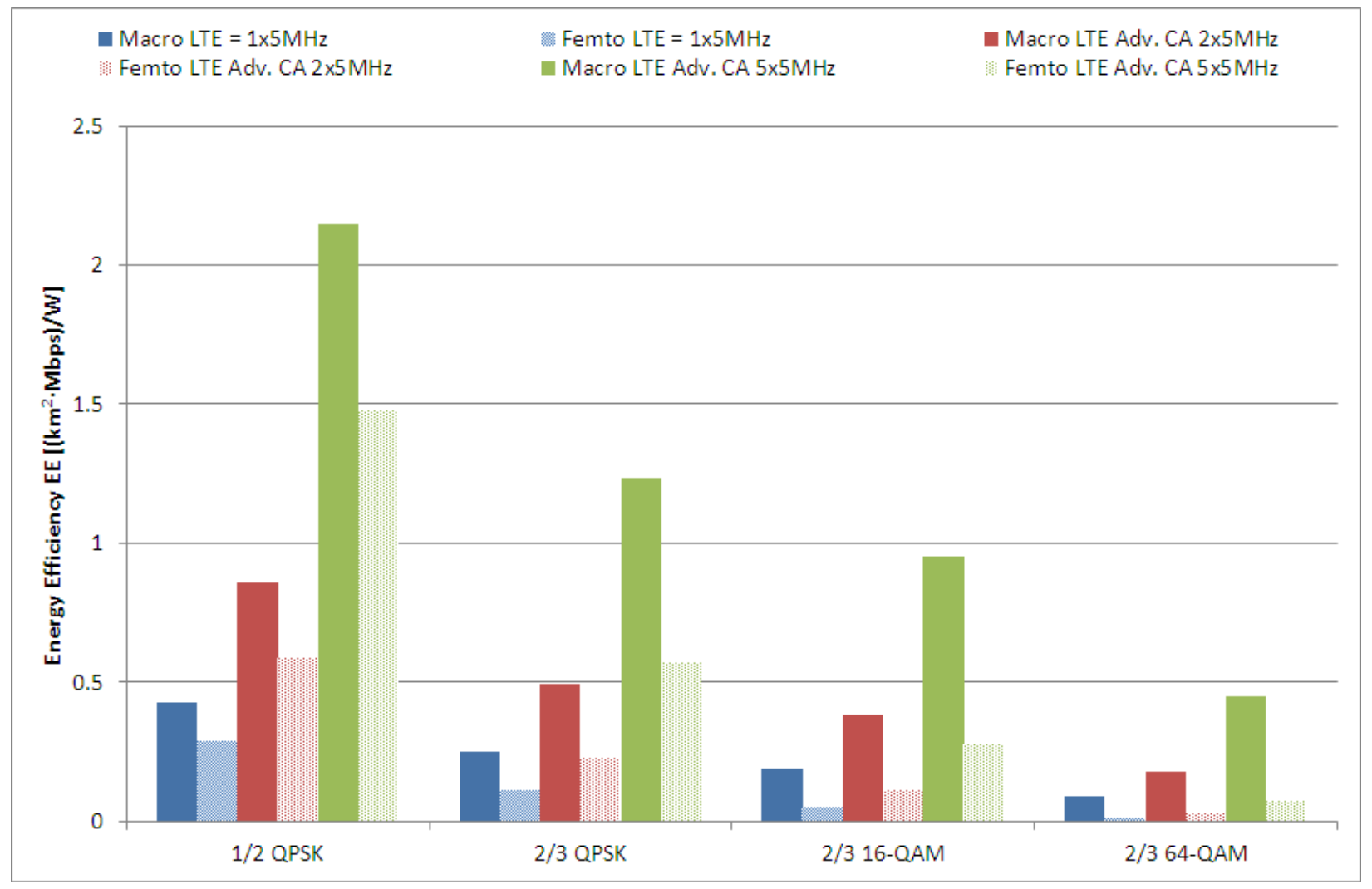

Figure 2. Energy efficiency EE of LTE and LTE-Advanced in a $5 \mathrm{MHz}$ channel.

network, without introducing Release 8/9. Similar results can be obtained for the other bandwidths.

\section{B. Heterogeneous deployments}

Based on Fig. 2, it can be concluded that a femtocell BS is less energy-efficient than a macrocell BS. However, this is not always the case.

In Fig. 3, the EE of an LTE-Advanced macrocell and femtocell BS is compared as a function of attainable bit rates. To plot Fig. 3, the bit rate was first defined for the aggregation of one to five component carriers with equal bandwidths. All possible bandwidths were considered. Next, for each possible bit rate and each BS type, the most energy-efficient solution was chosen, which is shown in Fig. 3.

Fig. 3 shows that it depends on the bit rate which BS type is the most energy-efficient. For bit rates higher than $20 \mathrm{Mbps}$, the macrocell BS is the most energy efficient $\left(E E=7.5\left(\mathrm{~km}^{2}\right.\right.$. $\mathrm{Mbps}) / \mathrm{W}$ versus $4.5\left(\mathrm{~km}^{2} \cdot \mathrm{Mbps}\right) / \mathrm{W}$ for $\left.25 \mathrm{Mbps}\right)$ due to its longer range and higher number of served users (even though its higher power consumption). Below $20 \mathrm{Mbps}$, there is no unambiguous answer. In some cases, the macrocell $\mathrm{BS}$ is most energy-efficient (e.g., for $5 \mathrm{Mbps}$ ) while in other cases the femtocell BS is most energy efficient (e.g., for $13 \mathrm{Mbps}$ ).

Due to carrier aggregation, high bit rates can be obtained even with a lower modulation scheme or bandwidth. E.g, $122 \mathrm{Mbps}$ is found by aggregation 3 carriers of $15 \mathrm{MHz}$ with 4/5 16-QAM, while $113 \mathrm{Mbps}$ is reached when aggregating 5 carriers of $20 \mathrm{MHz}$ with $2 / 3$ QPSK and $135 \mathrm{Mbps}$ by aggregating 4 carriers of $20 \mathrm{MHz}$ with 1/2 16-QAM. Due to the higher modulation scheme, the range is much lower for $122 \mathrm{Mbps}(141.3 \mathrm{~m})$ than for 133 and $122 \mathrm{Mbps}$ (respectively, $398.0 \mathrm{~m}$ and $327.2 \mathrm{~m}$ ), resulting in a lower $\mathrm{EE}$ which is responsible for the up-down behaviour of Fig. 3 .

It is obviously that future networks will consist of different BS types, although a good estimation of the needed bit rate, coverage and number of served users has to be made, to determine which type is the most suitable in each location of the network to reduce the network's power consumption. As demand varies over time, both BS types have to be deployed in the network and the optimal combination will results in a more energy-efficient network. This optimal combination will be obtained by first placing macrocell BSs to cover the area, followed by the femtocell BSs to foresee coverage in the socalled coverage holes i.e., the area's parts that are not covered by macrocell BSs. Furthermore, the capacity of the macrocell BSs can also be extended when necessary by using femtocell BSs in the coverage cell of the macrocell BSs.

\section{C. $M I M O$}

In this section, it is investigated how the extended support for MIMO influences the EE for spatial diversity and spatial multiplexing.

Fig. 4(a) shows the influence of spatial diversity on EE for different MIMO modes and both the macrocell and femtocell BS. A bit rate of $2.8 \mathrm{Mbps}$ in a $5 \mathrm{MHz}$ channel is considered. It is here assumed that the mobile station has the same number of receiving antennas as the BS has transmitting antennas which allows to determine the maximum EE gain [11]. SISO 


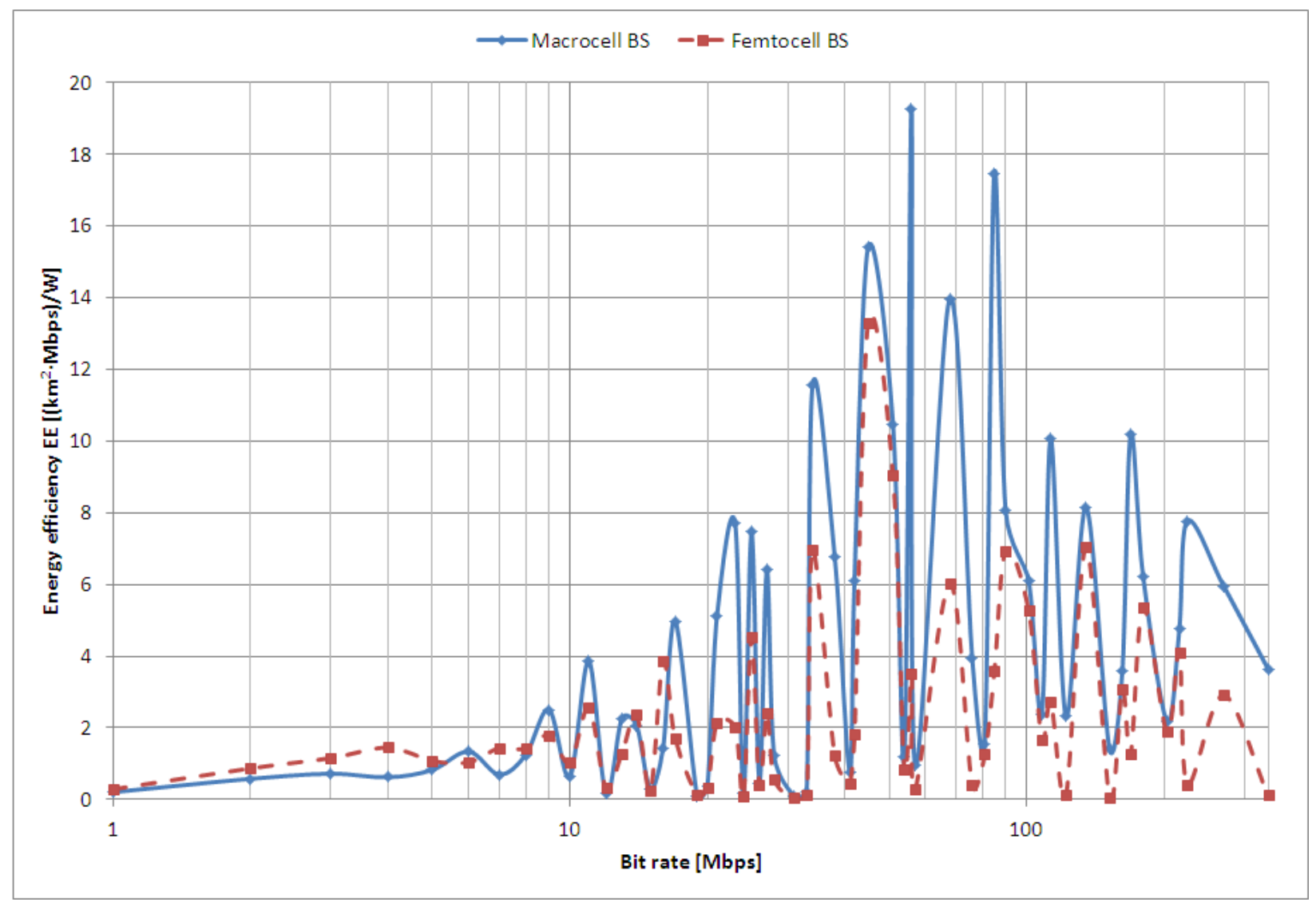

Figure 3. Comparison of a LTE-Advanced macrocell and femtocell base station for different bit rates.

(Single Input Single Output) i.e., only one transmitting and one receiving antenna, is used as reference scenario.

Fig. 4(a) shows that the higher the number of transmitting and receiving antennas, the higher the EE. For the macrocell $\mathrm{BS}$, the EE increases up to $433 \%$ when using $8 \times 8$ MIMO. In Eq. (1), $P_{e l}$ is 2 times higher, while $R$ is 3 times higher, resulting in a 5 times higher EE. For the femtocell BS, the EE increases even up to $454.6 \%$ (or 5.5 times).

Fig. 4(b) shows the results for spatial multiplexing. 1/3 QPSK and a $5 \mathrm{MHz}$ channel are considered. Again, a higher number of transmitting and receiving antennas results in a higher EE. For the macrocell BS, a maximum increase of $304.8 \%$ (or about 4 times) is found due to a 8 times higher bit rate while the power consumption increases only 2 times. For a femtocell BS, the EE gain is maximum $131.3 \%$ (or 2.5 times). The highest EE gain is obtained by using MIMO for spatial diversity.

For future networks, it is recommended to introduce MIMO, which is currently only in a limited way deployed. In addition to this, the highest possible MIMO mode should be used whenever these bit rates or ranges are needed.

\section{CONCLUSiON}

This study investigates how energy-efficient LTE-Advanced (Release 10) networks can be designed in comparison to LTE (Release 8/9) networks. Therefore, a power consumption model is developed for both macrocell and femtocell base stations and an appropriate energy efficiency measure is proposed. The influence of three main functionalities added to LTE-Advanced on the energy efficiency are investigated: carrier aggregation, heterogeneous networks, and MIMO.

In general, a higher bit rate result in a lower energy efficiency. However due to carrier aggregation, LTE-Advanced allows to obtain higher bit rates for even a higher energy efficiency.

Heterogeneous LTE(-Advanced) networks typically consists of macrocell and femtocell base stations. For bit rates above $20 \mathrm{Mbps}$, the macrocell base station is the most energy efficient. Below $20 \mathrm{Mbps}$, there is no unambiguous answer as it depends on the bit rate which one is the most energyefficient. For future networks, it is recommended to estimate accurately the required bit rate and coverage to decide which base station type or combination of these types should be used in the network.

Finally, MIMO can also increase the energy efficiency, even up to 5 times by using spatial diversity and $8 \times 8$ MIMO. Future networks should thus support MIMO.

In general, it is recommended that future networks implement LTE-Advanced as it will improve the energy efficiency compared to LTE.

\section{ACKNOWLEDGEMENT}

This work has been carried out with the financial support of the iMinds project 'Green Wireless Efficient City Access 


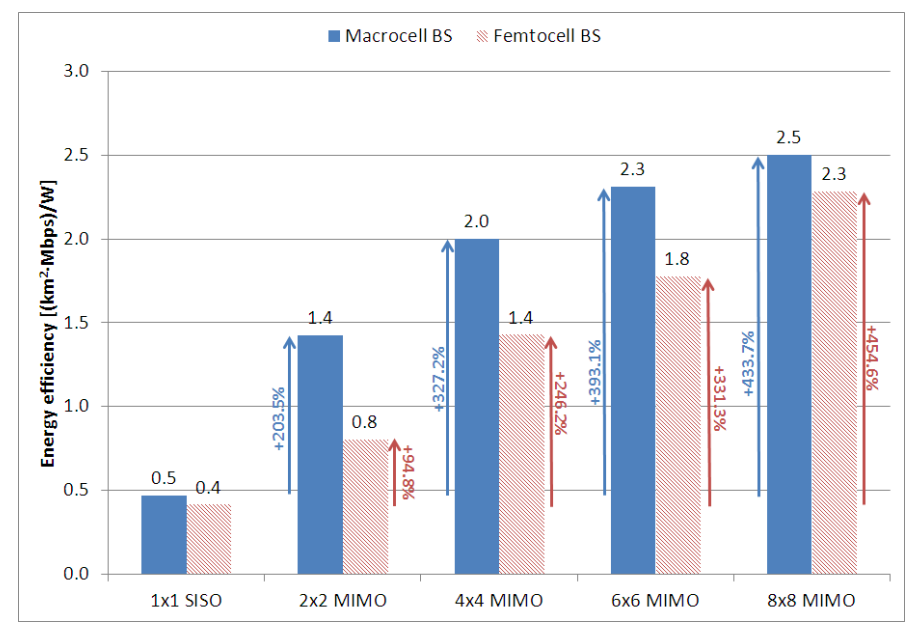

(a)

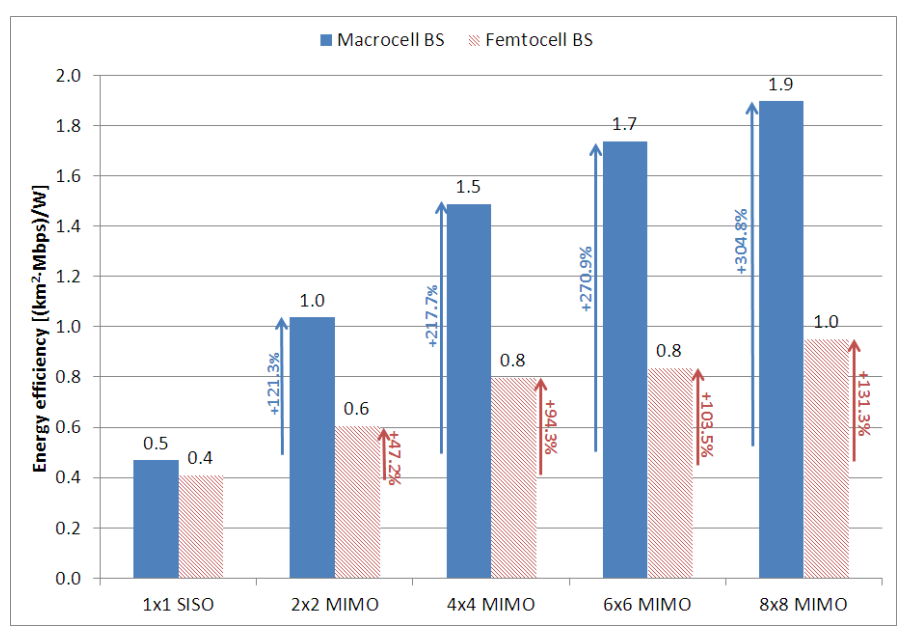

(b)

Figure 4. Influence of introducing MIMO (spatial diversity (a), spatial multiplexing (b)) on the energy efficiency EE for $1 / 3$ QPSK in a 5 MHz channel (\% shows the improvement compared to SISO, numbers on top indicate the $E E$-values).

Networks' (GreenWeCan).

W. Joseph is a Post-Doctoral Fellow of the FWO-V (Research Foundation Flanders).

\section{REFERENCES}

[1] M. W. Arshad, A. Vastberg, T. Edler, Energy Efficiency Improvement Through Pico Base Stations For A Green Field Operator, IEEE Wireless Communications and Networking Conference, 2012, pp. 2224-2229.

[2] M. Deruyck, W. Joseph, L. Martens, Power consumption model for macrocell and microcell base stations, Transactions on Emerging Telecommunications Technologies, doi: 10.1002/ett.2565, 2012.

[3] C. Khirallah, J. S. Thompson, D. Vukobratović, Energy Efficiency of Heterogeneous Networks in LTE-Advanced, IEEE Wireless Communications and Networking Conference, 2012, pp. 53-58.

[4] L. M. del Apio, E. Mino, L. Cucala, O. Moreno, I. Berberana, E. Torrecilla, Energy Efficiency and Performance in mobile networks deployments with femtocells, IEEE 22nd International Symposium on Personal Indoor and Mobile Radio Communications, 2011, pp. 107-111.

[5] 3GPP, 3rd Generation Partnership Project: Technical Specification Group Radio Access Network: Evolved Universal Terrestrial Radio Access (E-UTRA): User equipment (UE) Radio Transmission and Reception (TS 36.101 v8.17.0 Release 8, v9.1.0 Release 9, v10.1.0 Release 10), 2008-2011.

[6] E. Dahlman, S. Parkvall, J. Sköld, 4G LTE/LTE-Advanced for Mobile Broadband, ISBN:978-0-12-385489-6.

[7] L. M. Correia, D. Zeller, O. Blume, D. Ferling, Y. Jading, I. Gódor, G. Auer, L. Van der Perre, Challenges and Enabling Technologies for Energy Aware Mobile Radio Networks, IEEE Communications Magazine, Vol. 48, No. 11, 2010, pp. 66-72.

[8] Z. Niu, Y. Wu, J. Gong, Z. Yang, Cell Zooming for Cost-Efficient Green Cellular Networks, IEEE Communications Magazine, Vol. 48, No. 11, 2010, pp. 74-79.

[9] J. Baliga, R. Ayre, K. Hinton, R. S. Tucker, Energy Consumption in Wired and Wireless Access Networks, IEEE Communications Magazine, Vol. 49, No. 6, 2011, pp. 70-77.

[10] W. Vereecken, W. Van Heddeghem, M. Deruyck, B. Puype, B. Lannoo, W. Joseph, D. Colle, L. Martens, P. Demeester, Power Consumption in Telecommunication Networks: Overview and Reduction Strategies, IEEE Communications Magazine, Vol. 49, No. 6, 2011, pp. 62-69.

[11] M. Deruyck, E. Tanghe, W. Joseph, W. Vereecken, M. Pickavet, L. Martens, B. Dhoedt, Model for Power Consumption of Wireless Access Networks, IET Science, Measurement and Technology, Vol. 5, No. 4, 2011, pp. 155-161.

[12] I. Ashraf, F. Boccardi, L. Ho, Power Savings in Small Cell Deployments via Sleep Mode Technique, 21st Annual IEEE Symposium on Personal, Indoor and Mobile Radio Communications, 2010, pp. 356-361.

[13] M. Deruyck, D. De Vulder, W. Joseph, L. Martens, Modelling the Power Consumption in Femtocell Networks, IEEE Wireless Communications and Networking Conference, 2012, pp. 30-35.
Margot Deruyck was born in Kortrijk, Belgium on July 14, 1985. She received her M. Sc. degree in computer science engineering from Ghent University in July 2009. Since September 2009 she has been a research assistant in the Wireless and Cable (WiCa) research group at INTEC UGent/iMinds. Her scientic work is focused on green wireless access networks with minimal power consumption and minimal exposure of humans.

Wout Joseph received his M. Sc. degree in electrical engineering from Ghent University (Belgium) in July 2000. From September 2000 to March 2005, he was a research assistant at the Department of Information Technology (INTEC) of the same university. During this period, his scientific work was focused on electromagnetic exposure assessment. His research work dealt with measuring and modelling of electromagnetic fields around base stations for mobile communications related to the health effects of the exposure to electromagnetic radiation. This work led to a Ph. D. degree in March 2005. Since April 2005, he is postdoctoral researcher for iMinds-Ugent/INTEC. Since October 2007, he is a Post-Doctoral Fellow of the FWO-V (Research Foundation - Flanders). Since October 2009 he is professor in the domain of Experimental Characterization of wireless communication systems. His professional interests are electromagnetic field exposure assessment, propagation for wireless communication systems, antennas and calibration. Furthermore, he specializes in wireless performance analysis and Quality of Experience.

Bart Lannoo received a M.Sc. degree in electro-technical engineering and a Ph.D. degree from Ghent University (Belgium) in July 2002 and May 2008, respectively. Since August 2002, he has been working at the Internet Based Communication Networks and Services (IBCN) research group of the Department of Information Technology (INTEC) of Ghent University, where he is currently a postdoctoral researcher. As a member of the IBCN research group, he is also affiliated with iMinds. His research deals with fixed and wireless access networks, focusing on MAC protocols, Green ICT and technoeconomics. He has been involved in various national and European research projects, like the FP6 project IST-BREAD and the FP7 project ICT-ALPHA. Currently he is active in the European FP7 project ICT-OASE and coordinating the iMinds project GreenWeCan. 
Didier Colle received his M.Sc. degree in electrotechnical engineering (option: communications) from Ghent University in 1997. Since then, he has been working as a researcher in the IBCN research group at INTEC UGent/iMinds. His research led to a Ph.D. degree in February 2002. His work focuses on optical transport networks to support the next-generation Internet. $\mathrm{He}$ has been involved in several European projects like NOBEL, LASAGNE, and TBONES, and COST actions 266 and 291.

Luc Martens received the M.Sc. degree in electrical engineering from the Ghent University, Belgium in July 1986. From September 1986 to December 1990 he was a research assistant at the Department of Information Technology (INTEC) of the same university. During this period, his scientific work was focused on the physical aspects of hyperthermic cancer therapy. His research work dealt with electromagnetic and thermal modelling and with the development of measurement systems for that application. This work led to the Ph.D. degree in December 1990. Since 1991, he manages the wireless \& cable research group at INTEC. This group is since 2004 part of the iMinds institute and since April 1993 he is Professor at Ghent University. His experience and current interests are in modelling and measurement of electromagnetic channels, of electromagnetic exposure e.g. around telecommunication networks and systems such as cellular base station antennas, and of energy consumption in wireless networks. He is author or co-author of more than 300 publications in the domain of electromagnetic channel predictions, dosimetry, exposure systems and health and wireless communications. 